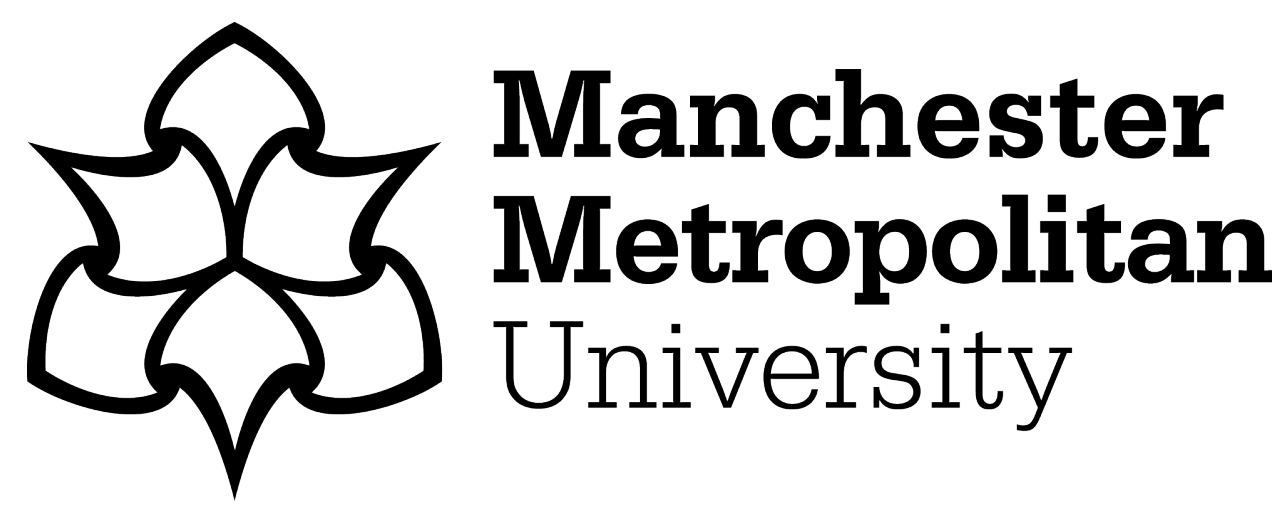

Rozman, M, Rabie, KM ORCID logoORCID: https://orcid.org/0000-00029784-3703 and Adebisi, B ORCID logoORCID: https://orcid.org/0000-00019071-9120 (2019) Wireless Power and Communication Transmission for Industrial Robots. In: 11th International Symposium on Communication Systems, Networks, and Digital Signal Processing (CSNDSP 2018), 18 July 2018 - 20 July 2018, Budapest, Hungary.

Downloaded from: https://e-space.mmu.ac.uk/620772/

Version: Accepted Version

Publisher: IEEE

DOI: https://doi.org/10.1109/CSNDSP.2018.8471796

Please cite the published version 


\title{
Wireless Power and Communication Transmission for Industrial Robots
}

\author{
Matjaz Rozman, Khaled M. Rabie, Bamidele Adebisi, \\ School of Engineering, Division of Electrical Engineering \\ Manchester Metropolitan University, M1 5GD UK \\ Emails:matjaz.rozman@stu.mmu.ac.uk; \{b.adebisi, k.rabie\}@mmu.ac.uk
}

\begin{abstract}
Mid-range resonant wireless power transfer (WPT) gained tremendous attention due to the higher efficiency. However, the relatively narrow transmission band has been simply drawn the technology back from being implemented in an application where the data and a power transmission are required. In this paper both the simulation and a practical implementation of a Strongly Coupled Magnetic Resonant (SCMR) system with a high frequency band for simultaneous data and power transmission is proposed. With this method communication and a power transmission can be transmitted in an industrial robot applications. Multiple $T_{X}$ and a single $R_{X}$ loop are placed between the joint of the robot in order to increase frequency spectre. The simulation has been developed in the theoretical analysis of equivalent circuits of the improved SCMR system. The analysis eventually leads to the factual parameters of a WPT system to provide its maximum efficiency for both wireless power and data transmission for an industrial robot. Besides the analysis has been validated with a comparison between the simulation results and practical implementation.
\end{abstract}

\section{INTRODUCTION}

Until 1921 "robot" was just a term limited to science fictions. Furthermore, fictions were turned into a reality by an English researcher William Grey Walter, creating the world's first autonomous robot with a complex behaviour in the late 1940s. With today's thriying technology, variety of robots such as droids, drones and robots are widely available for multiple purposes [1]. The introduction of the industrial robots replacing human work force, has resulted fivefold reduction in the defect rate, consistency, increased production and quality. Thus the robots are used to perform heavy duty, hazardous and repetitive work which is preferably not carried out by humans. According to IEEE.Org, it is also expected that by the end of 2018, China will have over 400,000 industrial robots, surpassing North America and the European Union in the number of robots operating in factories.

Three most widely utilised robot configurations for industrial automation can be listed as articulated robots, selective compliance assembly robot arm (SCARA) robots and gantry robots. Robots anatomy consists of joints and links to aid positioning and orientating, relatively accomplished by the inner (primal) joints and outer (distal) joints [2, 3]. However, the motion of an industrial robot is constrained by joint limits such as working angle due to the surrounded wires or the power supplying cables, as shown in Fig. 1A. An increment of the working angle of the robot as shown in Fig. 1B, will improve the speed and reduce the power consumption of the robot. The robot will no longer be limited by the working angle, moreover will be able to use the shortest distance towards the next move.

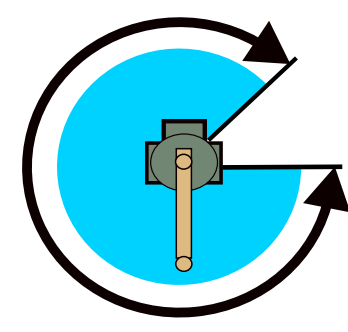

A



B
Fig. 1. Top view of (A) the work envelope of a typical SCARA horizontally articulated robot configuration and a robot configuration with SCMR technology (B)

Wireless Power Transfer (WPT) is another widely researched topic, which has been growing leaps and bounds in the last few years due to a sudden escalated awareness of wireless charging with the flourishing number of portable electronic components. By using WPT, all electronic devices will be capable of charging themselves, eliminating the conventional batteries or being plugged, which is hazardous, inconvenient and also simultaneously costly. Nicola Tesla became the first scientist to convince the WPT concept over his experiments, duplication of the work of Heinrich Hertz to power vacuum bulbs wirelessly in the early 20th century [4]. Nevertheless the experiment was not continued further due to the identified hazards, which were more likely to affect both the environment and the human factor.

A conventional SCMR system consists of two separate loops at close proximity $[5,6]$. In order to increase the transmission distance of the WPT system, both the transmitter and receiver loops must have the same oscillating frequency $[7,8]$. A maximum transmitting efficiency of the system can be achieved at the same frequency as the oscillating frequency of the loops. Numerous attempts have been made to enhance the frequency spectrum of the SCMR WPT system [9, 10]. For instance, the researchers in [11] have victoriously increased a frequency spectrum of comformal strongly resonant (CSCMR) WPT system. 


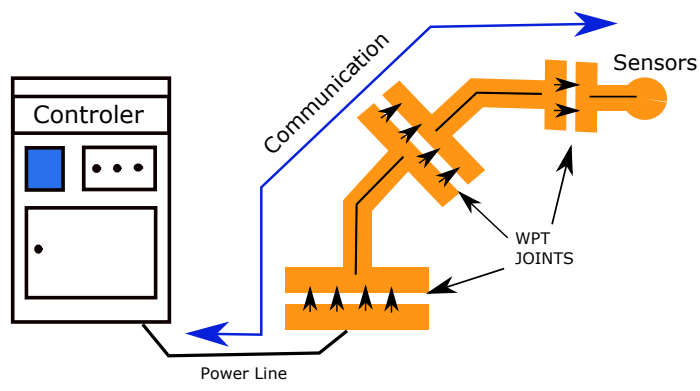

Fig. 2. Power and communication between the controller, robot and the sensor.

However, this paper focuses on the SCMR method, which is competent in transferring both power and data with high efficiency within short range, hence it is a more satisfactory solution to be implemented in robotic applications. In contrast with power transfer using slip ring, SCMR methor does not require friction between the two coils, thus does not induce noise into transmission channel [12, 13]. The frequency spectrum has been increased by connecting multiple coils in parallel with each other. Proposed method also reduces the complexity of the $T_{X}$ circuit, where only one resonant capacitor is used to tune the transmitter. Moreover, all the individual inductances in the transmitter coil are equvalent, but vary in size. This effort meant to enhance the flexibility of the industrial robots in many aspects, once implemented the degree of freedom angle will no longer be a crucial factor as presented in Fig. 2. The robot will be able move faster, thus will escalate the productivity while minimising the energy costs.

Initially, in section II a mathematical analysis of the proposed circuit has been illustrated. In section III practical implementation of the loops, is described. The results are explained in section IV, both the conclusion and the future work have been discussed in section $\mathrm{V}$.

\section{CIRCUIT ANALYSIS OF SCMR SYSTEM}

The electromagnetic resonance WPT concept is typically formed by two resonance coils: a transmitting resonator $\left(T_{X}\right)$ and a receiving resonator $\left(R_{X}\right)$, which resonate at the same frequency where their quality factor $(Q)$ is maximised. In a robot application, the coils may have the same diameter, which will simultaneously increase the transmission distance. Fig. 3 indicates the schematic of the proposed SCMR system with a multiple transmitter loop, embedded into the same plane. $R_{X}$ is represented by a single loop resonator while the distance between the two is represented by a coupling factor $(k)$, between them.
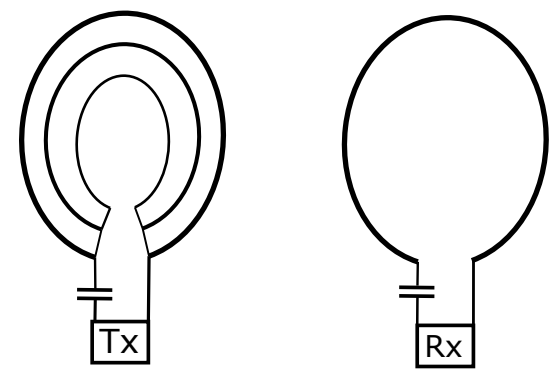

Fig. 3. Schematic model of the proposed SCMR system which enhances the frequency spectrum of the WPT for data transmission

Schematic diagram, Fig. 3 has been modelled into an equivalent circuit model as shown in Fig. 4, in order to represent how the proposed multi-loop SCMR system functions. $T_{X}$ side is represented by five independent inductance coils. Inductor $Z L_{1}$ together with the oscillating capacitor $Z C_{1}$, forms a power transmission loop. Inductors $Z L_{2}, Z L_{3}$, $Z L_{4}$ and $Z L_{5}$ are parallel connected set of inductors which are connected together with the capacitor $Z C_{2}$ in order to form the data transmission part of the WPT. This set of inductors, together forms a $T_{X}$ loop, in which every loop is connected to $R_{X}$ resonator by the coupling factor $k$. The $R_{X}$ loop is represented by an inductor $Z L_{6}$ and an oscillating capacitor $Z C_{3}$.

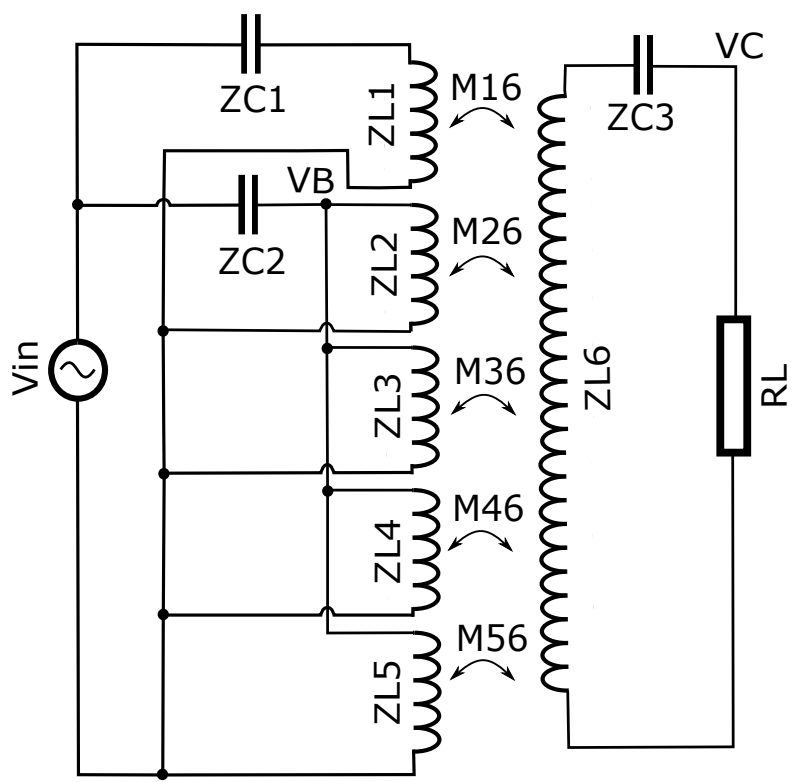

Fig. 4. Equivalent circuit of the CSCMR system with one pair of resonators

By applying Ampere's and Faraday's electromagnetic induction law, the magnetic induction field $\mathrm{H}$, from one of the two parallel coaxial loops at $d$, distance can be expressed as [14]

$$
H_{y}=\int \triangle H_{y}=\frac{I_{X} r_{y}^{2}}{2\left(r_{y}^{2}+d^{2}\right)^{\frac{3}{2}}}
$$

The mutual inductance of the two loops can be derived by including the area enclosed by the coil and can be written as 


$$
M_{x y}=\frac{\psi_{x y}}{I_{y}}=\frac{B_{y} A_{x}}{I_{y}}=\frac{\mu_{0} \pi r_{y}^{2} r_{x}^{2}}{2 \sqrt{\left(r_{y}^{2}+d^{2}\right)^{3}}}
$$

The angular frequency of the LC circuit can be calculated as $\omega=\frac{1}{\sqrt{L C}}$. However, in the proposed circuit the angular frequency can be calculated by taking into account each inductor connected in series as

$$
\omega=\frac{1}{\sqrt{\left(L_{1}+L_{2}+\ldots+L_{N}\right) C}}
$$

The circuit diagram in Fig. 4 can be solved by applying Kirchhoff's Voltage law (KVL) to the SCMR system. The currents $\left(I_{1} \sim I_{6}\right)$ in the circuit can be calculated as follows

$$
\begin{aligned}
& I_{1}=\frac{\left(V_{i n}-j \omega M_{16} I_{6}\right)}{\left(j \omega Z L_{1}\left(\frac{1}{j \omega C 1}\right)\right)} \\
& I_{2}=\frac{\left(V_{i n}-j \omega M_{26} I_{6}\right)}{j \omega Z L 2\left(\frac{1}{j \omega C_{2}}\right)} \\
& I_{3}=\frac{\left.\left(V_{i n}-j \omega M_{36} I_{6}\right)\right)}{j \omega Z L_{3}} \\
& I_{4}=\frac{\left(V_{i n}-j \omega M_{46} I_{6}\right)}{j \omega Z L_{4}} \\
& I_{5}=\frac{\left(V_{B}-j \omega M_{56} I_{6}\right)}{j \omega Z L_{5}} \\
& I_{6}=-\frac{V_{C}+A}{j \omega Z L_{6} \frac{1}{j \omega C_{6}}}
\end{aligned}
$$

Where $A=\left(I_{5} j \omega M_{56}\right)+\left(I_{4} j \omega M_{46}\right)+\left(I_{3} j \omega M_{36}\right)+$ $\left(I_{2} j \omega M_{26}\right)+\left(I_{1} j \omega M_{16}\right)$ and $M_{x}$ is the mutual inductance between the ith and jth loop. Considering voltages on separate branches a gain on the output can be calculated as:

$$
(G)=V_{C}\left((B-D) *(j w)^{2}+(E-F) j w+G+H\right)+R_{L}(B)
$$

where

$$
\begin{gathered}
\left.A=\left((J+K) V_{i n}(j w)+N V_{i n}+(O+P) V_{B}\right)\right) \\
B=-Z C_{2} Z C_{6} Z L_{1} Z L_{3} Z L_{4} Z L_{5} M_{26}^{2} \\
D=Z C_{1} Z C_{6} Z L_{2} Z L_{3} Z L_{4} Z L_{5} M_{16}^{2} \\
E=-Z C_{6} Z L_{1} Z L_{2} Z L_{3} Z L_{4} M_{56}^{2} \\
F=Z C_{6} Z L_{1} Z L_{2} Z L_{3} Z L_{5} M_{46}^{2} \\
G=Z C_{6} Z L_{1} Z L_{2} Z L_{3} Z L_{4} Z L_{5} R_{L} \\
H=Z L_{1} Z L_{2} Z L_{3} Z L_{4} Z L_{5} Z L_{6} \\
J=-Z C_{2} Z C_{6} Z L_{1} Z L_{3} Z L_{4} Z L_{5} M_{26}^{2} \\
K=Z C_{1} Z C_{6} Z L_{2} Z L_{3} Z L_{4} Z L_{5} M_{16}^{2} \\
N=-Z C_{6} Z L_{1} Z L_{2} Z L_{3} Z L_{5} M_{46} \\
O=Z C_{6} Z L_{1} Z L_{2} Z L_{3} Z L_{4} M_{56} \\
P=Z C_{6} Z L_{1} Z L_{2} Z L_{4} Z L_{5} M_{36}
\end{gathered}
$$
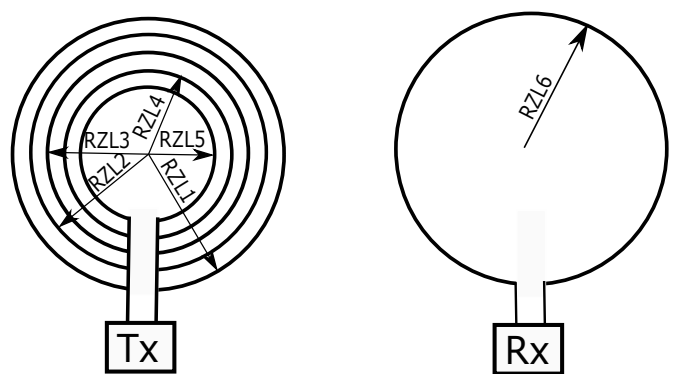

Fig. 5. Transmitter loops and receiver loop diameters

\section{CIRCUIT ANALYSIS}

The resonant loops have been designed as a circle shape since the robot joints make circular motions where the oval shaped coils provides maximum alignment at any position of the robot arm. $T_{X}$ coils have been placed concentrically inside same plane to minimize its size. The diameter of the loops varies according to the calculated coupling factor between the $T_{X}$ and $R_{X}$ loops. Thus the circuit is designed to demonstrate high efficiency at coupling factor between power transmitter loop and a receiver at 0.5 . The rest of the coils are arranged in the way that their coupling factor with Tx coils decreased to 0.1 . The circuit has its maximum power transmission efficiency at the frequency of $1.02 \mathrm{MHz}$, where the efficiency reaches $97.8 \%$.

The cross section radius of the resonant loops, as presented in Fig. 5, can be analytically calculated, applying to the equation (2) however, it can be adjusted according to the robot size. The cross section of the power transmission loops for this experiment, can be calculated as $10.2 \mathrm{~cm}$ while data transmission loops cross section warius from $2.1 \mathrm{~cm}$ to $9.4 \mathrm{~cm}$, depends on the $k$ factor required. Similarly the capacitance required for the loop oscillator can be calculated using (3). Similar to $Z C_{1}, Z C_{2}$ is calculated as $680 \mathrm{pF}$. The radius of $T_{X}$ loop can be calculated using (3) which is equal to $10.2 \mathrm{~mm}$. Based on the calculations, the frequency spectrum of the transmitter increase by adding additional inductors into the circuit. As represented in Fig. 6 a frequency spectrum of the system with single transmitting loop is very narrow. When an additional inductor is added the frequency spectrum increases, and the efficiency is higher than $50 \%$ for frequency band from $1 \mathrm{MHz}$ to $5 \mathrm{MHz}$. With a third coil added, the frequency band increases further, the efficiency, higher than $50 \%$ is reached for a spectrum from $1.3 \mathrm{MHz}$ to $8 \mathrm{MHz}$, while maximum efficiency of $92 \%$ is reached at frequency of $1 \mathrm{MHz}$. 


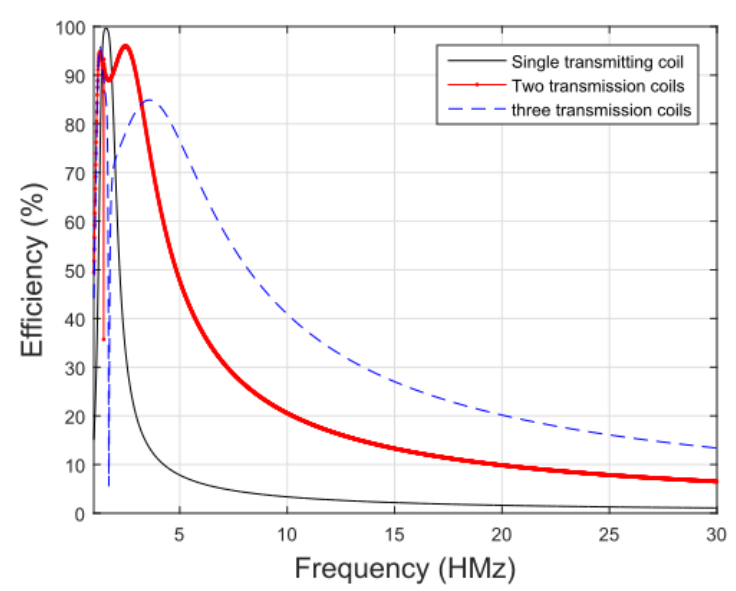

Fig. 6. Comparison of the frequency range between the circuit with two transmitting coils and circuit with three transmitting coils.

Fig. 7 represents the calculated results for a system with a single transmitting loop and a proposed multiple transmitter loop. The analysis illustrates the maximum efficiency of the single loop is for $2 \%$ higher that the one with multiple loops with $98 \%$. The frequency spectrum of the system with multiple loops is higher than $50 \%$ for a frequency from $200 \mathrm{kHz}$ to $1.6 \mathrm{MHz}$ and $2.3 \mathrm{MHz}$ up to $16 \mathrm{MHz}$. The circuit is able to transmit power with the efficiency of 97 , and transmit the data with efficiency as high as $68 \%$. Enhanced frequency spectrum allows the sensors embedded in the robot, to communicate and send the relative crucial information for its functionality.

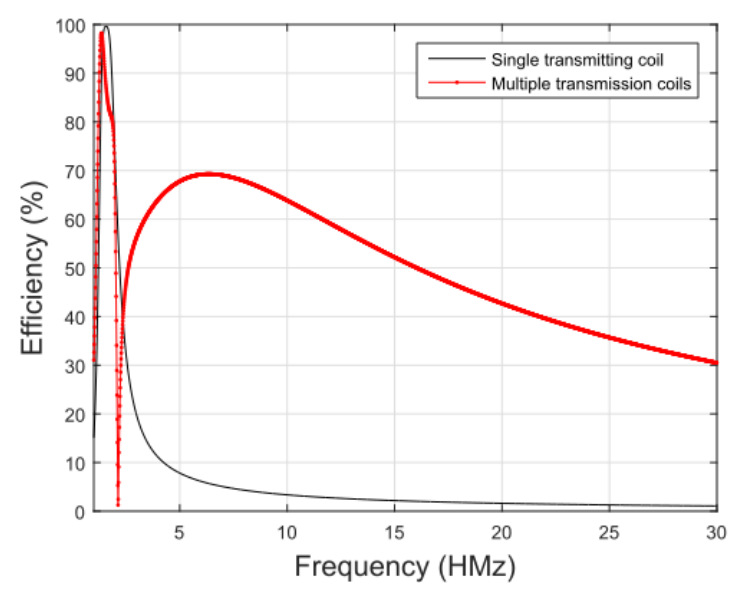

Fig. 7. Comparison between the system with single transmitter coil and the proposed transmitter with multiple coils.

In Table II calculated Transmitter and Receiver loop components are presented.

TABLE I

Calculated elements

\begin{tabular}{|c|c|}
\hline Transmitter & Receiver \\
\hline \hline$Z C 1, Z C 3=680 p F$ & $C R_{X}=650 p F$ \\
\hline$L T_{X} 1, L T_{X} 2, L T_{X} 3, L T_{X} 4$ and $L T_{X} 5=16.37 \mu H$ & $L R_{X}=19.65 \mu H$ \\
\hline
\end{tabular}

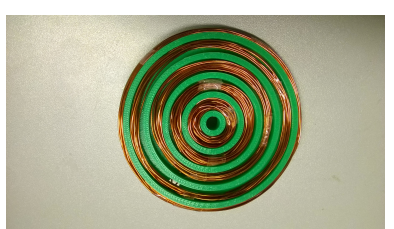

(a) Transmitter coil structure design contains a multiple coils in same plane.

Fig. 8. Milti-coil transitter used in thos experiment (a) and measurement set up (b).

\section{RESULTS}

In this section a result of the practical implementation is compared to the calculated model. In Fig. 8A a practical implementation of a Transmitter loops can be seen. The model contains five loops which are embedded into same plane, however the distance between them varies depending on calculated $\mathrm{k}$ required. The coils have been placed inside a plastic housing, in order to secure the position. In Fig. 8B the measurements set-up can be seen. A $T_{X}$ coil is placed close to the $R_{X}$ coil. The power is send between the two coils and a efficency of the transmission between them is measured. A signal generator was used to provide power to the $T_{X}$, while the results were measured by usb based spectrum analyser.

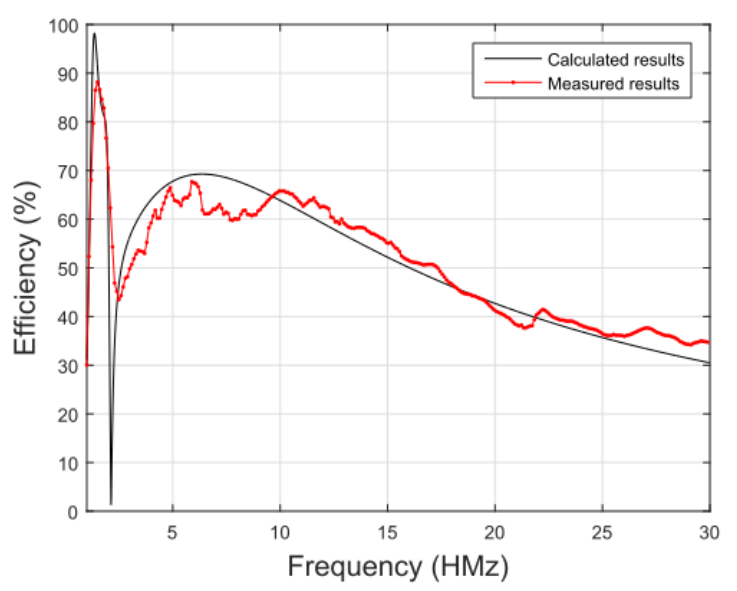

Fig. 9. measurements

The results of the measurements are presented and compared to the calculated values in Fig. 9. It can be seen that the measured curve follows the calculated values, however the measured power transmission efficiency is with 87 for $11 \%$ bellow calculated value. A data transmission side reaches 50 efficiency at a frequency of $2.5 \mathrm{MHz}$ and falls below that value at $17.6 \mathrm{MHz}$, which form a $15.1 \mathrm{MHz}$ frequency band. The measured frequency spectrum is $1.3 \mathrm{MHz}$ above calculated value. Overall measured results confirm that the proposed model is able to increase the frequency band of the SCMR WPT system to enable simultaneously power and data transmission. 


\section{Conclusions}

This paper represents an approach of applying the SMRC in robotic applications, where the distance between the transmitter and the receiver stays constant. The system has been designed to transmit both power and data from the transmitter side to the receiver. A mathematical model has been developed to calculate the theoretical efficiency of the transmission based on the coupling factor between milti-coil transmitter and single receiver coil. Calculated results are compared with prototype model. Replacing a conventional power supply for an SCMR based wireless power supply will not only increases the degree of freedom, but also the flexibility to control the robot manipulator from a distant location. Results show that the frequency spectrum of SCMR increase from 2 to $17.1 \mathrm{MHz}$, enabling high efficiency power transmission and create a $15.1 \mathrm{MHz}$ communication channel. Efficiency of data transmission varies from maximum 50 to minimum $69 \%$.

Future work will be to extend the distance between the transmitter and receiver while increasing the frequency bandwidth of the signal for higher transmission speed of data. However the SCMR based WPT along with PLC based robot applications could be further customized to suit the industry needs via Industry 4.0.

\section{ACKNOWLEGMENT}

This work was funded by TSB as part of the "Smart InBuilding Micro Grid for Energy Management" (Innovate UK project 101836) .

\section{REFERENCES}

[1] M. A. Kamel, X. Yu, and Y. Zhang, "Fault-tolerant cooperative control design of multiple wheeled mobile robots," IEEE Trans. on Control Syst. Tech., vol. 26, no. 2, pp. 756-764, March 2018.

[2] M. Garduño-Aparicio, J. Rodríguez-Reséndiz, G. Macias-Bobadilla, and S. Thenozhi, "A multidisciplinary industrial robot approach for teaching mechatronics-related courses," IEEE Trans. on Education, vol. 61, no. 1, pp. 55-62, Feb 2018.

[3] P. Neto, J. N. Pires, and A. P. Moreira, "Accelerometerbased control of an industrial robotic arm," in 18th IEEE Internat. Symp on Robot and Human Interactive Commun., Toyama, Japan, Sept 2009, pp. 1192-1197.

[4] Y. Wang and W. Chen, "A novel design method of rf lens for long-range wireless power transmission," IEEE Antennas and Wireless Propag. Letters, vol. 16, pp. 3159-3162, 2017.

[5] D. Liu and S. V. Georgakopoulos, "Misalignment study of cylindrical scmr wireless power transfer system," in 2016 IEEE Internat. Symp. on Antennas and Propag. (APSURSI), Fajardo, Puerto Rico, June 2016, pp. 413-414.

[6] Z. N. Low, R. A. Chinga, R. Tseng, and J. Lin, "Design and test of a high-power high-efficiency loosely coupled planar wireless power transfer system," IEEE Trans. on Industrial Electronics, vol. 56, no. 5, pp. 1801-1812, May 2009.
[7] Y. Bonan, K. Bao, C. Flores, K. Baza, and S. V. Georgakopoulos, "Efficiency analysis of a conformal scmr system for wearable devices," in 2017 IEEE Internat. Symp. on Antennas and Propag. USNC/URSI Nat.l Radio Science Meeting, San Diego, CA, USA, July 2017, pp. 1233-1234.

[8] I. Ghotbi, M. Najjarzadegan, H. Sarfaraz, S. J. Ashtiani, and O. Shoaei, "Enhanced power-delivered-toload through planar multiple-harmonic wireless power transmission," IEEE Trans. Circuits and Syst. II: Express Briefs, vol. PP, no. 99, pp. 1-1, 2018.

[9] Y. Wang, Y. Yao, Y. Guan, X. Liu, M. Liu, and D. Xu, "A novel modulation and demodulation method for wireless power and data transmission," in 2017 IEEE Transportation Electrification Conf. and Expo, AsiaPacific (ITEC Asia-Pacific), Harbin, China, Aug 2017, pp. 1-6.

[10] W. Xu, T. Cheng, C. Wu, N. Lu, Z. Yang, Y. Lian, and G. Wang, "Ultrathin flexible coils for wireless power and data link in biomedical sensors," in 2017 IEEE 12th Internat. Conf. on ASIC (ASICON), Guiyang, China, Oct 2017, pp. 604-607.

[11] H. Hu and S. V. Georgakopoulos, "Multiband and broadband wireless power transfer systems using the conformal strongly coupled magnetic resonance method," IEEE Trans. on Ind. Electr., vol. 64, no. 5, pp. 3595-3607, May 2017.

[12] G. He, Q. Chen, X. Chen, and P. Xin, "An accurate modeling method for electric parameters prediction of contactless slip ring," in 2017 IEEE Energy Conversion Congress and Exposition (ECCE), Oct 2017, pp. 2051-2056.

[13] J. Zhang, X. Ma, B. Cheng, and Y. Zhao, "Storage life modeling and analysis for contacting slip ring based on physics of failure," IEEE Transactions on Components, Packaging and Manufacturing Technology, vol. 7, no. 12, pp. 1969-1980, Dec 2017.

[14] R. Inoue, D. Miyagi, M. Tsuda, and H. Matsuki, "High-efficiency transmission of a wireless power transmission system for low-frequency using rebco double-pancake coils," IEEE Trans. on Applied Superconductivity, vol. 27, no. 1, pp. 1-6, Jan 2017. 